\title{
BMJ Open Effectiveness of physical therapy in addition to occlusal splint in myogenic temporomandibular disorders: protocol of a randomised controlled trial
}

\author{
Cristina Incorvati (D) , ${ }^{1}$ Antonio Romeo, ${ }^{2}$ Adele Fabrizi, ${ }^{1}$ Luca Defila, ${ }^{1}$ Carla Vanti, ${ }^{2}$ \\ Maria Rosaria Antonella Gatto, ${ }^{3}$ Claudio Marchetti, ${ }^{1}$ Paolo Pillastrini ${ }^{2}$
}

To cite: Incorvati C, Romeo A, Fabrizi A, et al. Effectiveness of physical therapy in addition to occlusal splint in myogenic temporomandibular disorders: protocol of a randomised controlled trial. BMJ Open 2020;10:e038438. doi:10.1136/ bmjopen-2020-038438

- Prepublication history for this paper is available online. To view these files, please visit the journal online (http://dx.doi. org/10.1136/bmjopen-2020038438).

Received 12 March 2020 Revised 13 May 2020

Accepted 17 June 2020

\section{Check for updates}

(c) Author(s) (or their employer(s)) 2020. Re-use permitted under CC BY-NC. No commercial re-use. See rights and permissions. Published by BMJ.

${ }^{1}$ Department of Biomedical and Neuromotor Sciences (DIBINEM) Oral and Maxillofacial Surgery, Alma Mater Studiorum University of Bologna, Bologna, Emilia-Romagna, Italy

${ }^{2}$ Department of Biomedical and Neuromotor Sciences (DIBINEM) Phisical Therapy, Alma Mater Studiorum University of Bologna, Bologna, Emilia-Romagna, Italy ${ }^{3}$ Department of Biomedical and Neuromotor Sciences(DIBINEM) Medical Statistics, Alma Mater Studiorum University of Bologna, Bologna, Emilia-Romagna, Italy

Correspondence to

Dr Cristina Incorvati, cristina.incorvati@unibo.it

\section{ABSTRACT}

Introduction Temporomandibular disorders (TMDs) are considered a collection of musculoskeletal conditions involving the masticatory muscles, the temporomandibular joint and associated structures. The myogenous group appears to represent the most frequently diagnosed category. In the context of a multimodal approach, splint therapy and musculoskeletal physiotherapy are often considered as a preferred therapy. The purpose of this study will be to investigate the effects of musculoskeletal physiotherapy combined with occlusal splint and education versus occlusal splint and education alone in the treatment of chronic myogenous TMD on pain and mandibular range of motion.

Methods and analysis All consecutive adults complaining of TMDs presented to the Department of Biomedical and Neuromotor Sciences of the University of Bologna will be considered eligible. Inclusion criteria shall be based on the presence of myogenous TMDs, as diagnosed through clinical examination in reference to the international diagnostic criteria of TMDs. Randomisation, concealed allocation, blinded assessment and intention-to-treat analysis will be employed. The splint therapy will consist of the use of the splint every night and concurrent delivery of an educational programme; the protocol shall have a duration of three consecutive months. The combined musculoskeletal physiotherapy, splint therapy and education will additionally consist of manual therapy techniques and exercise; such protocol shall consist of a duration of three consecutive months, inclusive of 10 sessions for the enhanced elements. All outcome measures will be collected at baseline, after treatment and at a 6 months follow-up.

Ethics and dissemination Ethical approval has been obtained from the Independent Ethic Committee in Clinical Research of AUSL Bologna-Italy (47/2018/SPER/ AUSLB0). Pursuant to applicable rules,we will obtain informed consent from each participant and collect data anonymously to maintain privacy. Results will be disseminated to clinicians and researchers through peerreviewed publications and conferences.

Trial registration number NCT03726060

\section{INTRODUCTION}

Temporomandibular disorders (TMDs) are considered a collection of musculoskeletal
Strengths and limitations of this study

- This study concerns a pragmatic monocentric singleblind, concealed and randomised clinical trial, with two arms including multimodal therapeutic intervention in primary healthcare; the application of physical therapy shall be the main contrast between intervention and control groups.

- Doctors and physical therapists who will be involved in the project shall receive specialised clinical training to manage the selected patient profile according to the international guideline diagnostic criteria for temporomandibular disorders.

- The effectiveness of the intervention will be evaluated through validated and objective outcome measures of pain and temporomandibular range of motion.

- The effectiveness of the two protocols will also be affected by the participants' compliance in accordance with the instructions received; it will be impossible to determine and measure the participants' adherence to such instructions.

- On commencement of the study, participants shall be administered at baseline the Hospital Anxiety and Depression Scale test; however, other than the aforementioned self-assessment, no other psychometric data will be collected during the course of the clinical trial

conditions involving the masticatory muscles, the temporomandibular joint (TMJ) and associated structures, ${ }^{1}$ and are the main cause of pain of non-dental origin in the orofacial region including head, face and related structures. ${ }^{2}$ Symptoms of TMDs include decreased mandibular range of motion (ROM), TMJ pain, TMJ joint sounds with function, myogenous pain and functional limitation or deviation during jaw opening. ${ }^{3-5}$ TMDs are frequently associated with other symptoms; by example, neck pain, ear-related symptoms, musculoskeletal impairment of the cervical spine $^{6-10}$ and headache. Individuals with myofascial TMD are significantly more likely 
to suffer from chronic daily headache, migraine and tension-type headaches in comparison with individuals without TMD pain. ${ }^{11}$

Epidemiological studies indicate that approximately $10 \%-15 \%$ of the general population has TMDs, and within such group $5 \%$ of the respondents require therapy. The highest prevalence of TMDs is found in subjects between 18 and 45 years of age. Among female gender during the childbearing years ${ }^{12}$; it concerns specifically chronic TMDs. ${ }^{14}$

Among the different categories of TMDs, the myogenous group presents the highest frequency (42\%), followed by disc displacement with reduction $(32.1 \%)$, arthralgia $(30 \%)$, osteoarthrosis $(14.2 \%)$, osteoarthritis $(12.3 \%)$ and disc displacement without reduction $(8.6 \%) .{ }^{15}$ These findings have been confirmed in a recent meta-analysis including 21 epidemiological studies wherein the prevalence, according to the Research Diagnostic TMD Criteria, was for the muscle disorder group $45.3 \%$, for the disc displacements group $41.1 \%$ and the for joint disorders group $30.1 \%$, respectively. ${ }^{16}$ Although the aetiology of TMDs is still not fully understood, it is largely considered to be multifactorial. The focus of aetiological theories on TMDs has shifted from peripheral to central factors. Current understanding and evidence-based literature fail to demonstrate a direct relation between the 'historical' occlusal factors and TMDs signs and symptoms. Presently, a minor role is assigned to natural dental occlusion as a risk factor for TMDs in favour to central factors, for example, psychological factors, pain sensitivity and genetics. ${ }^{1}$ Frequency of somatic symptoms (such as runny nose, fatigue and dizziness), general psychological symptoms, negative mood, symptoms of post-traumatic stress and stress emerged as important risk factors for incident TMD pain. ${ }^{14} 17$ It has been reported that subjects with muscle TMD pain tend to show higher prevalence of mood and panic/agoraphobic symptoms than the remaining groups. ${ }^{18}$ Additionally, there is a significant association among TMD pain and poor sleep quality, ${ }^{14} 19$ self-reports of jaw parafunction, ${ }^{14}$ whiplash trauma, ${ }^{20}$ and peripheral and central sensitisation mechanism. ${ }^{21}$

TMD diagnosis is mainly based on a combination of defined signs and symptoms. The most accepted and worldwide used diagnostic criteria are the validated diagnostic criteria for TMD (DC/ TMD). ${ }^{4}$ These include Axis I diagnostic criteria derived from pertinent clinical TMD signs and symptoms and Axis II consisting of psychosocial and behavioural questionnaires. Axis I diagnostic criteria for TMD pain-related disorders have acceptable validity and provide definitive diagnoses for pain involving the TMJ, masticatory muscles and headache attributed to TMD4. Nevertheless, more than one of these diagnoses seem to be present in $35.2 \%$ of the patients. ${ }^{15}$ It is important to note that the DC/TMD definitions of myogenous pain (type I) may be potentially confusing, as such include pain in the jaw, temple, ear or in front of the ear and disc displacements; bony changes and joint effusion are also common findings. ${ }^{22}$ Therefore, on a clinical setting, it is challenging to identify pure muscle pain patients; this could explain the conflicting findings often found in previous TMDs trials. Current studies suggest that management of TMDs should be aimed at decreasing pain, decreasing loading on the muscles and joints, and facilitating patients' restoration of function and quality of life. ${ }^{23} \mathrm{~A}$ recent systematic review recommends conservative, not invasive and reversible therapies; occlusal adjustments are not considered an appropriate modality for TMDs treatment, being irreversible, not scientific based form of therapy. ${ }^{1}$ A multimodal approach is considered the proper management in myogenous TMDs including: counselling, acupuncture, exercises, occlusal splint therapy and manual therapy. In case of severe acute pain or chronic pain, pharmacotherapy and minimally invasive procedures can be included. ${ }^{24}$

An exercise regimen seems to provide moderate shortterm and varying long-term benefits in pain reduction and ROM improvement in patients with TMDs, but seems to be lacking a significant impact for functional improvement. ${ }^{25}$ Musculoskeletal manual approaches seem to be effective for treating TMD. A recent meta-analysis ${ }^{26}$ showed a significant difference and large effect on active mouth opening and on pain during active mouth opening in favour of musculoskeletal manual techniques when compared with other conservative treatments for TMD, and these effects are greater in the short term.

The effects of the different manual therapy procedures, such as myofascial release and massage techniques applied on the masticatory muscles, result in more effective outcomes as compared with control groups (low to moderate evidence); nevertheless, those approaches are statistically as effective as toxin botulinum injections (moderate evidence). Upper cervical spine thrust manipulation or mobilisation techniques are more effective compared with control groups (low to high evidence), while thoracic manipulations are not. ${ }^{27}$ However, no high-quality evidence was found. ${ }^{28}$

Occlusal splints (OSs) are the most popular treatment for TMDs. Investigators described various types of occlusal splints with different designs, indications and functions. Nevertheless, the most common design is the full-arch, flat-plane maxillary stabilisation splint. Occlusal splint therapy can provide centric relation occlusion, eliminate posterior interferences, provide anterior guidance on anterior teeth, reduce neuromuscular activity and establish stable occlusal relationships with uniform tooth contacts throughout the dental arch. Nevertheless, the mechanism of action of occlusal splints is still unknown. ${ }^{29}$ The most widely recognised application of OSs for treating TMDs is their use in patients with myogenous diagnosis according to DC/TMD classification.

In most of the studies, positive results were obtained with both a real OS and a placebo control, but in many of these studies the real appliance was superior. ${ }^{22}$ The conclusion of a recent meta-analysis, which focused on the effects of hard stabilisation appliances in myogenous TMD subjects, was that there is qualitatively poor but 
significant evidence that flat plane occlusal appliances are more effective than non-occluding appliances when worn during the night only. ${ }^{30}$ OSs have been reported to be effective in pain reduction, decreasing muscle tenderness in the short term; nevertheless, their effect is equalised with other therapeutic modalities in long-term follow-up. ${ }^{31}$

Most of the previous clinical trials compared different conservative treatments, but no clinical study to date has investigated the effects of a multimodal approach, consisting in a combination of musculoskeletal physiotherapy, education and occlusal splint.

The primary objective of this randomised controlled trial will be to compare the effectiveness of adding musculoskeletal physiotherapy to occlusal splint and education versus occlusal splint and education alone in the treatment of chronic myogenous TMD within the context of short-term follow-up. The secondary objective will be to evaluate variation in mandibular ROM and to investigate the presence of any psychosocial factors.

\section{METHODS}

\section{Study design}

This study is a double-arm randomised controlled trial in a 1:1 allocation ratio with concealed randomisation and triple blinding according to a parallel design.

\section{Study settings and population}

All consecutive adult patients (18 years and older) complaining of TMDs addressed to the Department of Biomedical and Neuromotor Sciences of Bologna University (Italy) will be eligible for the clinical trial. An oral and maxillofacial surgeon $(\mathrm{AF})$, with substantive training and experience in TMDs diagnosis will perform a clinical and functional examination of each patient according to diagnostic criteria for TMDs (DC/TMD). ${ }^{4}$

\section{Eligibility criteria}

The criterion for inclusion in the study shall be existence of myogenous pain, by provocation test inquiring as to local myalgia, myofascial pain and myofascial pain with referral in accordance with the DC/TMD. ${ }^{4}$ Each accepted patient should report a history of ongoing pain, either transient or constant, for a duration of more than 3 months, have good knowledge of spoken and written Italian language, and provide informed consent, as evidenced by a signed document.

The following factors shall cause candidates to be excluded from the study: edentulism to a degree which makes application of the occlusal splint impossible, history of severe neurological disorders, articular and muscular autoimmune diseases, active malignant neoplasm presence of psychiatric conditions, history of alcoholism, drug and pain medication abuse, inclusion in other experimental protocols, patients undergoing surgical and/or radiation therapy on the cervical and facial areas, other TMDs without a myogenous component, other treatments of the cervical and TMJ areas in the previous 3 months, use of drugs which can affect neuromuscular system and/or pregnancy.

\section{Interventions}

Patients shall be allocated according to the study's protocol among these parallel groups: occlusal splint therapy and education (control group), and musculoskeletal physiotherapy combined with occlusal splint therapy and education (test group).

\section{Occlusal splint therapy}

Participants in each group will receive a maxillary splint. The maxillary splint used in this study will be the stabilisation/Michigan splint consisting of a rigid splint constructed for the maxillary arch, including all maxillary teeth, with a flat occlusal plane. ${ }^{22}$

Following the indication of the clinician (CI), a technician will make the occlusal splint with methyl methacrylate acrylic, with maximum $2 \mathrm{~mm}$ occlusal thickness. During the delivery of the splint, the clinician will adjust the device, so that the opposing dentition will occlude uniformly, evenly and simultaneously with the occluding surface of the splint in centric occlusion. Splint retention will be provided by coverage of the labial and buccal surfaces of the maxillary teeth with the addiction of ball metal claps, if necessary.

For all participants, CI will take accurate alginate impressions of both arches and an interocclusal wax wafer registration. After 1 week, participants will receive the occlusal splint appropriately adjusted in the centric occlusion. The splint therapy will consist in the overnight use of the splint for 3 months with specific educational instructions through an informative brochure.

\section{Education}

The educational programme shall consist of delivery of educational advice through an informative brochure providing suggestions for the avoidance of disuse, abuse, overuse of the TMJ and on the correct use of occlusal splint (box 1).

\section{Physical Therapy}

Physical treatment shall consist of a series of interventions: teaching of self-treatment techniques to be performed by the patient at home and manual therapy techniques addressed to temporomandibular area, cervical spine and cervicothoracic junction. Each session will be carried out individually and will have a duration of $45 \mathrm{~min}$. This time period will be divided as follows: 25 min dedicated to the temporomandibular area, $15 \mathrm{~min}$ to the cervical and cervicalthoracic junction, and $5 \mathrm{~min}$ for teaching self-treatment techniques to be carried out at home and methods to verify correct performance (table 1). Musculoskeletal physiotherapy programme will consist of 10 sessions distributed over 3 months.

During the entire study period, no participants will receive any other form of treatment, including drugs or occlusal adjustments. Occlusal splint and educational 
Box 1 Educational booklet

\section{Anatomy of the temporomandibular joint}

Temporomandibular joint is formed by the mandibular fossa of the temporal bone and the mandibular condyle that fits into it. In between those bones, there is an articular disc.

Both the condyle and the mandibular fossa are covered by a dense layer of fibrocartilaginous tissue, which primarily allows the joint to resist loads during function, and to reduce attrition between the bony surfaces.

Even though it is formed by these two bones, temporomandibular joint is considered to be a compound joint as the articular disc function as a third bone. The articular disc is composed of dense fibrous connective tissue, with few blood vessels or nerve fibers Box 1for the most part (only the extreme periphery of the disc is slightly innervated).

Posteriorly the articular disc is attached to the retrodiscal tissue, an area of connective tissue that is highly vascularised and innervated, and that is composed by collagenous fibres and elastic fibres.

The superior and inferior parts of the anterior region of the disc are attached to the capsular ligaments, which surround the joint. The articular disc is attached anteriorly, posteriorly, medially and laterally to these ligaments.

There can be found two distinct cavities limited by them: the upper one is bordered by the mandibular fossa and the superior part of the disc, the lower one by the condyle and the superior part of the disc.

On the internal surfaces of these cavities, there is a lining of endothelial cells known as the synovial lining: it produces the synovial fluid, which fills the cavities nourishing the non-vascular surfaces. It also acts as a lubricant medium to minimise friction.

\section{Temporomandibular joint disorders}

Each of the anatomical structures described can be involved in pathological processes, characterised by signs and symptoms, like: pain at rest or chewing, sounds like pops or clicks and limited range of motion.

Temporomandibular disorders affect $10 \%-25 \%$ of the population. The international classification divides these disorders into articular (arthralgias), muscular (myalgias) and those concerning the articular disc (dislocations or displacement). In particular, in myalgias, muscle tissue is responsible for painful symptoms, which can be perceived in the muscle itself or even in distant parts (eg, at the level of the teeth, in the ear or in the head).

Neck pain and headache are often associated with temporomandibular disorders.

\section{Treatment}

The treatment of these disorders should be conservative. The most used therapies include anti-inflammatory and analgesic drugs, the use of an intraoral splint (also known as occlusal bite) and physiotherapy (which includes muscle manipulation and exercises). Scientific research has shown that these therapeutic solutions are useful in the short and long term.

For the success of those treatments, it is of great importance to add behaviours that favour the reduction or correction of the loads on the joint, muscles and the articular disc.

Below is a list of some recommended changes which are easy to adopt and have proven to be helpful in people who suffer from temporomandibular disorders:

Try not to bite your nails.

Try not to bite your lower lip.

- Try not to grit your teeth but keep the two dental arches slightly separate. To help doing this, try to keep your tongue resting on the upper palate, at the point of the palate that is touched when we pronounce the letter $\mathrm{N}$.

Try not to consume chewing gum,even occasionally.

- Try not to nibble pencils or ballpoint pens.

intervention will be conducted by the same clinician whereas musculoskeletal physiotherapy treatment will be performed by an orthopaedic manipulative physical therapist (AR), expert in this field.

\section{Outcomes}

The primary outcome measure will be the subjective perception of pain. A Visual Analogue Scale (VAS), represented by a $10 \mathrm{~cm}$ long scale with a score from 0 to 10 , will be used to measure the intensity of perceived pain. The VAS has been shown to be a reliable ${ }^{32}$ and valid ${ }^{33}$ instrument for measuring pain intensity in acute and chronic patient. Its minimal clinically important difference for general chronic pain is $30 \%$ of pain reduction ${ }^{34}$ and for pain related to TMD is $1.2 \mathrm{~cm}$ on the maximum pain, 1.9 $\mathrm{cm}$ on the current pain and $0.9 \mathrm{~cm}$ on the minimum pain scales. ${ }^{35}$ As it has been suggested, the above-described assessment of acute pain during movement (dynamic pain) is more important than pain at rest, ${ }^{36}$ therefore,
VAS data will be collected during three different clinical evaluations: at rest, during maximum mouth opening and during chewing. The chewing test will be performed while the patient is chewing gum.

The secondary outcome measures shall concern TMJ ROM and psychological factors. ROM is the most important measure for TMJ function, providing analytical and millimetric measurement of functional movements. ROM measure will be collected using a precision electrical gauge positioned between the incisal edges of the upper and lower central incisor teeth.

In addition to maximum oral opening, a complete gnathological examination will be performed for each patient and specific parameters will be recorded, including: mandibular deviation from the midline, left and right lateral excursion movements, protrusive movements, overjet and overbite. The reliability of these measures has been shown ranging from Intraclass 
Table 1 Musculoskeletal physiotherapy

\subsection{Manual therapy}

a. Temporomandibular joint
Traction mobilisation in resting or specific
position

\begin{tabular}{|c|c|}
\hline & $\begin{array}{l}\text { Lateral glide mobilisation in resting } \\
\text { position }\end{array}$ \\
\hline & Anterior glide mobilisation \\
\hline \multicolumn{2}{|l|}{ b. Cervical spine } \\
\hline & Posterior glide occiput-C1 (mobilisation) \\
\hline & $\begin{array}{l}\text { Unilateral postero-anterior C1 } \\
\text { (mobilisation) }\end{array}$ \\
\hline & $\begin{array}{l}\text { Unilateral postero-anterior C2 } \\
\text { (mobilisation) }\end{array}$ \\
\hline & Inferior glide C2 (mobilisation) \\
\hline & Superior glide C2 (mobilisation) \\
\hline & Cervicothoracic junction (manipulation) \\
\hline \multicolumn{2}{|l|}{ c. Soft tissues } \\
\hline & $\begin{array}{l}\text { Myofascial release or trigger } \\
\text { point compression directed to the } \\
\text { following muscles (where positive to } \\
\text { palpation test): masseter, temporalis, } \\
\text { pterigoideus medialis and lateralis, } \\
\text { sternocleidomastoid, suprahyoid } \\
\text { muscles, semispinalis, multifidus, } \\
\text { suboccipitalis, splenius capitis and } \\
\text { cervicis and levator scapulae }\end{array}$ \\
\hline
\end{tabular}

\subsection{Self-treatment}

Self-massage of tender or trigger points in the above-mentioned muscles. 2', 2 times a day, starting from the first treatment

Temporomandibular joint selfmobilisation. 2', 2 times a day, starting from the first treatment

Neurodynamic self-mobilisation (opening the mouth in craniocervical flexion). 40 movements (with flexed elbow)+40 movements (with supinated forearm and extended elbow, wrist and fingers), starting from the third treatment

Craniocervical flexion exercise. 10"x10, 2 times a day, starting from the fourth treatment

Deep cervical extensors exercise. 10 " $x 10,2$ times a day, starting from the fourth treatment

Coefficient Correlation (ICC) $=0.70-0.9837$ and from $\mathrm{k}=0.40-0.8938$, with an excellent reliability for vertical mandibular motions and poor to moderate reliability for excursive movements. ${ }^{37} 38$

Moreover, a qualitative assessment of passive end feel will be collected (eg, painful, hard, elastic, etc) with a score abbreviation ( $\mathrm{p}=$ painful, $\mathrm{H}=$ hard, $\mathrm{E}=$ =lastic) .
Psychological factors will be measured using the Hospital Anxiety and Depression Scale (HADS) in its Italian version. ${ }^{39}$ HADS is a questionnaire used to identify the presence of anxiety and depression in people with non-psychiatric health problems. It is divided into two subscales, one on anxiety (HADS-A) and one on depression (HADS-D), each containing seven mixed items. Each item is rated on a scale from 0 to 3 , with a maximum score of 11 for each subscale. ${ }^{40}$ An aggregate score of 11 or more indicates a significant case of psychiatric comorbidity, a score of 8-10 means the presence of a disorder, a score of 7 or less is considered normal. A cutoff point of $8 / 21$ gives a specificity of 0.78 and a sensitivity of 0.90 for anxiety (HADS-A) and a specificity of 0.79 and a sensitivity of 0.83 for depression (HADS-D) ${ }^{41}$ HADS has been shown to be a valid and reliable measure of overall emotional distress in patients with chronic pain. ${ }^{42}$

A second examiner (LD), who was masked as to patients' treatment, will perform the baseline measure of primary and secondary outcomes and independently collect data at the end of treatment ( 3 months after the start) and at 6 months after the start of treatment. Patients' timeline is shown in figure 1. In order to improve patients' compliance and adherence to the study, the researchers will adopt the following strategies: written instructions for both occlusal splint and exercises, and gnathological recall visits at 18 weeks after the start of each treatment.

\section{Adverse events}

Participants will be asked to report any side effects of treatments (eg, fatigue, stiffness, muscle spasm or increased pain). In the eventa side effect is reported, participants will be asked to rate its degree of severity, from light to severe. Moderate and severe side effects will be considered the rules to leave the study (stopping rules).

\section{Sample size}

Sample size was based on pain considered the primary outcome; endpoint of pain was the VAS commonly accepted for pain measurements.

At an $\alpha$ level of 0.05 with a power equal to 0.80 hypothesising that the difference on pain between the two treatments was $\geq 1.9 \mathrm{~cm}$ and by using as estimate the current pain according to Calixtre et $a l^{35}$ a minimum of 26 subjects per group needed.

\section{Randomisation}

Allocation of patients in one of the two parallel treatment groups will be based on a randomisation list. Randomisation will be performed using a random, computer-generated, numeric sequence provided by the biostatistician (MRAG) and placed in sealed opaque envelopes. Researchers' assistants will enrol and assign participants to their respective groups.

\section{Blinding}

This trial will be triple blinded: participants, independent assessor (LD), biostatistician (MRAG). All outcome measures will be collected at baseline, after treatment (3 


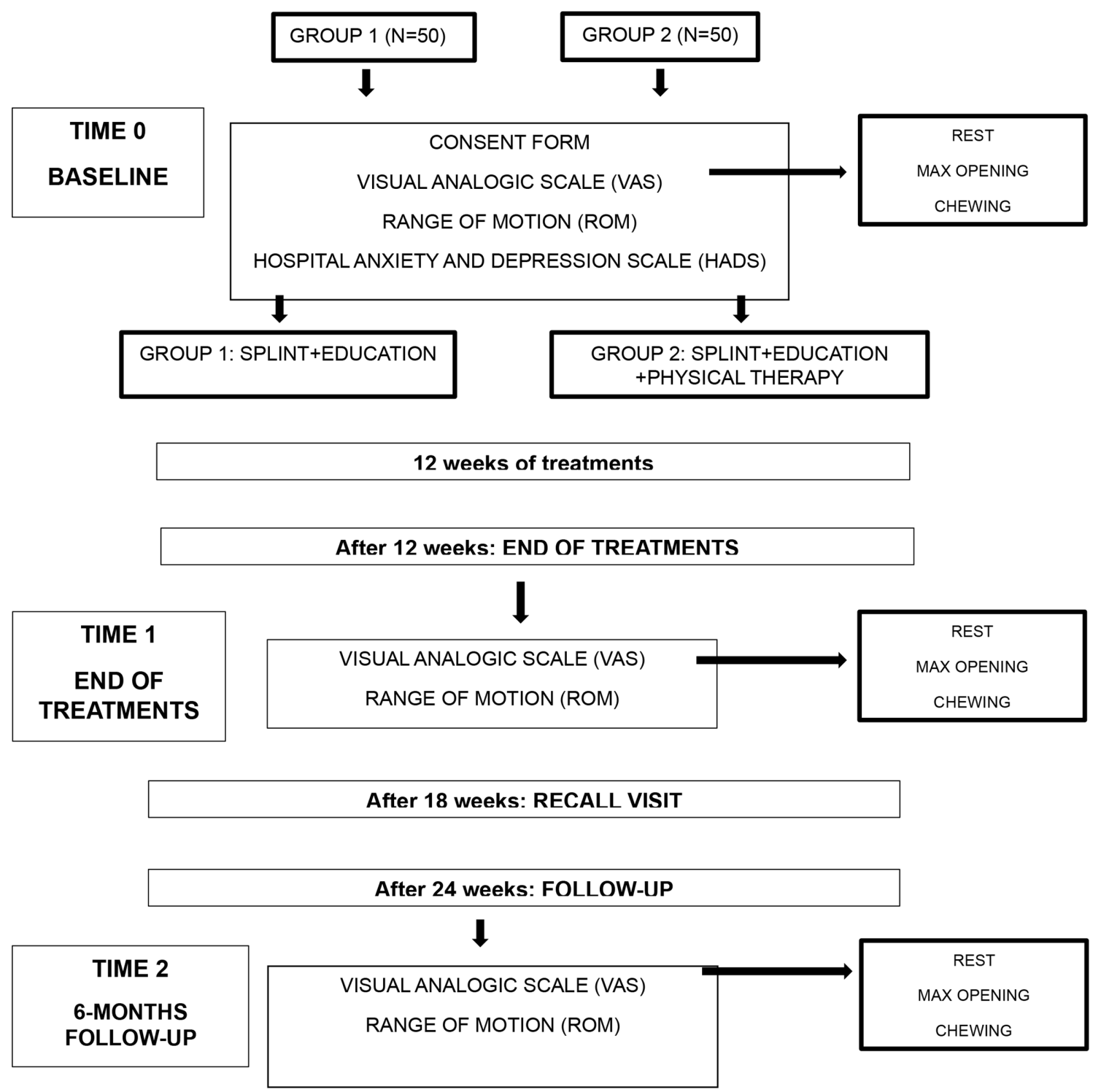

Figure 1 Flow chart of the trial.

months from the beginning of the treatment) and at 6 months follow-up by the same LD, who will be blinded to group allocation of each participant.

\section{Statistical methods}

Trial statistician (MRAG) will conduct all analyses and all reporting following Consolidated Standards of Reporting Trials guidelines. Primary analysis will be based on the 'intention-to-treat' population of all randomised participants regardless of their compliance with the protocol in order to handle missing data. Quantitative variables will be synthetised by means of arithmetic mean and SD or median and IQR after verifying the Gaussian distribution by using the Shapiro-Wilks test. Categorical variables will be synthetised by means of univariate and bivariate frequency distributions. Explorative analysis of the questionnaire will be performed by means of multiple correspondence analysis.

Comparison between the two groups will be performed by means of t-test or Mann-Whitney U-test based on distribution of VAS (primary outcome) and ROM (secondary outcome). As for HADS cluster analysis will be carried out aiming to evidence possible groupings of items corresponding to the two arms of treatment. Generalised linear model (mixed-effects model) will be applied aiming to evaluate the influence of treatment, demographic factors, clinical factors and their interactions on the primary and secondary outcomes. $\alpha$ level will be a priori set at 0.05 for a two tails test.

\section{Data management}

Each subject participating in the trial will be uniquely identified by a number, and information such as name, address will be maintained in a separate database. When forms on outcome measures will be completed by the investigator, data will be reported in an Excel datasheet without any identification concerning group allocation. Data on adverse events (type, severity, etc) will be also reported in the same Excel datasheet. Data verification, validation, maintenance and updating will be maintained 
in a password-protected database under the control of the statistician.

\section{Patient and public involvement}

This study shall be undertaken due to our clinical observations concerning good results of a multimodal and multidisciplinary approach (consisting in a combination of musculoskeletal physiotherapy, education and occlusal splint) in patients complaining of TMDs. Despite the major clinical advantages of this therapeutic approach, the authors noticed that there is no robust evidence due to the lack of large randomised trials in the field.

As a consequence, patients do not receive clear indications on the most effective treatment options. With the exception of the research question, patients were not involved in the design of the study neither in the dissemination plan of our research. The burden of the trial was not assessed before commencement.

At completion of the study, all patients will be informed about the general findings of the study through a summarised handbook.

\section{ETHICS AND DISSEMINATION}

The study protocol was approved by the Independent Ethic Committee in Clinical Research of AUSL Bologna-Italy (protocol number 47/2018/SPER/ AUSLBO- 21/02/2018).

The procedures will be conducted according to the Declaration of Helsinki. A researchers' assistant will obtain a written informed consent from all participants before they will enter the study. Privacy of the patients will be respected, and identification of the included subjects will be made by a unique code, consisting of a progressive number. We will adopt strategies to spread our results by peer-reviewed publications, congress presentations and dissemination to policy-makers.

\section{DISCUSSION}

TMDs are one of the most burdensome musculoskeletal disorders in the adult population and are often associated with psychological discomfort, physical disability and functional limitations in the orofacial system. Given the social and economic consequences of this condition, it is relevant to find the most effective conservative treatments to reduce pain and improve function. This large randomised controlled trial will compare the effects of two different conservative approaches in the management of patients with TMDs: musculoskeletal physiotherapy in addition to occlusal splint and education, versus occlusal splint and education alone. The results will have an influence on the conservative treatment prescription and the clinical decision-making process.

Acknowledgements The authors thank Laboratorio D-entity and Mr Roberto Accorsi for providing Occlusal Splints. The authors thank all patients, who partecipate in the trial for their contributions of valuable data. The authors thank Mrs Andretta Lucille Stevens, English editor, for her great help in the editing revsion.
Contributors Conceptualisation: AF, Cl, PP, AR and CV. Data curation: LD. Formal analysis: MRAG. Investigation: AF, Cl, AR and CV. Methodology: AF, Cl, PP, AR and CV. Resources:software:supervision: $\mathrm{CM}$ and PP. Writing-original draft: $\mathrm{Cl}, \mathrm{CM}, \mathrm{PP}, \mathrm{AR}$ and CV. Writing-review and editing: AF, Cl, CM, PP, AR, CV and MRAG.

Funding The authors have not declared a specific grant for this research from any funding agency in the public, commercial or not-for-profit sectors.

Competing interests None declared.

Patient and public involvement Patients and/or the public were not involved in the design, or conduct, or reporting, or dissemination plans of this research.

Patient consent for publication Not required.

Provenance and peer review Not commissioned; externally peer reviewed.

Open access This is an open access article distributed in accordance with the Creative Commons Attribution Non Commercial (CC BY-NC 4.0) license, which permits others to distribute, remix, adapt, build upon this work non-commercially, and license their derivative works on different terms, provided the original work is properly cited, appropriate credit is given, any changes made indicated, and the use is non-commercial. See: http://creativecommons.org/licenses/by-nc/4.0/.

ORCID iD

Cristina Incorvati http://orcid.org/0000-0002-2759-7930

\section{REFERENCES}

1 Rinchuse DJ, Greene CS. Scoping review of systematic review Abstracts about temporomandibular disorders: comparison of search years 2004 and 2017. Am J Orthod Dentofacial Orthop 2018;154:35-46.

2 Leeuw R, Glasser G. American Academy of Oral Facial Pain. Diagnosis and management of TMDs. In: De Leeuw R, Glasser G, eds. Orofacial pain: guidelines for classification, assessment, and management 5th ED. Chicago Quintessence Publ Co, 2013.

3 Peck CC, Goulet J-P, Lobbezoo F, et al. Expanding the taxonomy of the diagnostic criteria for temporomandibular disorders. J Oral Rehabil 2014;41:2-23.

4 Schiffman E, Ohrbach R, Truelove E, et al. Diagnostic criteria for temporomandibular disorders (DC/TMD) for clinical and research applications: recommendations of the International RDC/TMD Consortium Network* and orofacial pain special interest group. J Oral Facial Pain Headache 2014;1:1-27.

5 Schiffman E, Ohrbach R. Executive summary of the diagnostic criteria for temporomandibular disorders for clinical and research applications. J Am Dent Assoc 2016;147:438-45.

6 Graff-Radford SB, Abbott JJ. Temporomandibular disorders and headache. Oral Maxillofac Surg Clin North Am 2016;28:335-49.

7 Visscher CM, Lobbezoo F, de Boer W, et al. Prevalence of cervical spinal pain in craniomandibular pain patients. Eur $\mathrm{J}$ Oral Sci 2001;109:76-80.

8 de Wijer A, de Leeuw JR, Steenks MH, et al. Temporomandibular and cervical spine disorders. self-reported signs and symptoms. Spine 1996;21:1638-6646.

9 Grondin F, Hall T, Laurentjoye M, et al. Upper cervical range of motion is impaired in patients with temporomandibular disorders. Cranio 2015;33:91-9.

10 Silveira A, Gadotti IC, Armijo-Olivo S, et al. Jaw dysfunction is associated with neck disability and muscle tenderness in subjects with and without chronic temporomandibular disorders. Biomed Res Int 2015:2015:512792

11 Gonçalves DAG, Camparis CM, Speciali JG, et al. Temporomandibular disorders are differentially associated with headache diagnoses: a controlled study. Clin J Pain 2011;27:611-5.

12 Macfarlane TV, Glenny AM, Worthington HV. Systematic review of population-based epidemiological studies of oro-facial pain. J Dent 2001;29:451-67.

13 Magnusson T, Egermark I, Carlsson GE. A longitudinal epidemiologic study of signs and symptoms of temporomandibular disorders from 15 to 35 years of age. J Orofac Pain 2000;14:310-9.

14 Slade GD, Ohrbach R, Greenspan JD, et al. Painful temporomandibular disorder: decade of discovery from OPPERA studies. J Dent Res 2016;95:1084-92.

15 Poveda-Roda R, Bagan JV, Sanchis JM, et al. Temporomandibular disorders. A case-control study. Med Oral Patol Oral Cir Bucal 2012;175:e794-800.

16 Manfredini D, Guarda-Nardini L, Winocur E, et al. Research diagnostic criteria for temporomandibular disorders: a systematic 
review of axis I epidemiologic findings. Oral Surg Oral Med Oral Pathol Oral Radiol Endod 2011;112:453-62.

17 Fillingim RB, Ohrbach R, Greenspan JD, et al. Psychological factors associated with development of TMD: the OPPERA prospective cohort study. J Pain 2013;14:T75-90.

18 Manfredini D, Bandettini di Poggio A, Cantini E, et al. Mood and anxiety psychopathology and temporomandibular disorder: a spectrum approach. J Oral Rehabil 2004;31:933-40.

19 Riley JL, Benson MB, Gremillion HA, et al. Sleep disturbance in orofacial pain patients: pain-related or emotional distress? Cranio 2001;19:106-13.

20 Häggman-Henrikson B, List T, Westergren HT, et al. Temporomandibular disorder pain after whiplash trauma: a systematic review. J Orofac Pain 2013;27:217-26.

21 Fernández-de-las-Penas C, Svensson P. Myofascial temporomandibular disorder. Curr Rheumatol Rev 2016;12:40-54.

22 Greene CS, Menchel HF. The use of oral appliances in the management of temporomandibular disorders. Oral Maxillofac Surg Clin North Am 2018;30:265-77.

23 Kandasamy S, Greene CS, Rinchuse DJ, et al. Tmd and Orthodontics: a clinical guide for the Orthodontist 1st ED. Switzerland: 2015 Springer International Publishing, 2015.

24 Wieckiewicz M, Boening K, Wiland P, et al. Reported concepts for the treatment modalities and pain management of temporomandibular disorders. J Headache Pain 2015;16:106.

25 Dickerson SM, Weaver JM, Boyson AN, et al. The effectiveness of exercise therapy for temporomandibular dysfunction: a systematic review and meta-analysis. Clin Rehabil 2017;31:1039-48.

26 Martins WR, Blasczyk JC, Aparecida Furlan de Oliveira M, et al. Efficacy of musculoskeletal manual approach in the treatment of temporomandibular joint disorder: a systematic review with metaanalysis. Man Ther 2016;21:10-17.

27 Calixtre LB, Moreira RFC, Franchini GH, et al. Manual therapy for the management of pain and limited range of motion in subjects with signs and symptoms of temporomandibular disorder: a systematic review of randomised controlled trials. J Oral Rehabil 2015;42:847-61.

28 Armijo-Olivo S, Pitance L, Singh V, et al. Effectiveness of manual therapy and therapeutic exercise for temporomandibular disorders: systematic review and meta-analysis. Phys Ther 2016;96:9-25.

29 Michelotti A, lodice G, Vollaro S, et al. Evaluation of the shortterm effectiveness of education versus an occlusal splint for the treatment of myofascial pain of the jaw muscles. J Am Dent Assoc 2012;143:47-53.
30 Delsnyder J, Colina T, Elsemary N, et al. Stabilization appliances as treatment for myogenous temporo- mandibular disorders: a systematic review and meta-analysis. Open Journal of Dentistry and Oral Medicine 2017:5:72-84.

31 Kuzmanovic Pficer J, Dodic S, Lazic V, et al. Occlusal stabilization splint for patients with temporomandibular disorders: meta-analysis of short and long term effects. PLoS One 2017;12:e0171296.

32 Bijur PE, Silver W, Gallagher EJ. Reliability of the visual analog scale for measurement of acute pain. Acad Emerg Med 2001;8:1153-7.

33 Gaston-Johansson F. Measurement of pain: the psychometric properties of the Pain-O-Meter, a simple, inexpensive pain assessment tool that could change health care practices. $J$ Pain Symptom Manage 1996;12:172-81.

34 Dworkin RH, Turk DC, Wyrwich KW, et al. Interpreting the clinical importance of treatment outcomes in chronic pain clinical trials: IMMPACT recommendations. J Pain 2008;9:105-21.

35 Calixtre LB, Oliveira AB, Alburquerque-Sendín F, et al. What is the minimal important difference of pain intensity, mandibular function, and headache impact in patients with temporomandibular disorders? clinical significance analysis of a randomized controlled trial. Musculoskelet Sci Pract 2020;46:102108.

36 Breivik $\mathrm{H}$, Borchgrevink PC, Allen SM, et al. Assessment of pain. Br J Anaesth 2008;101:17-24.

37 Dworkin SF, LeResche L, DeRouen T, et al. Assessing clinical signs of temporomandibular disorders: reliability of clinical examiners. $J$ Prosthet Dent 1990;63:574-9.

38 Leher A, Graf K, PhoDuc J-M, et al. Is there a difference in the reliable measurement of temporomandibular disorder signs between experienced and inexperienced examiners? J Orofac Pain 2005; 19:58-64.

39 Costantini M, Musso M, Viterbori P, et al. Detecting psychological distress in cancer patients: validity of the Italian version of the hospital anxiety and depression scale. Support Care Cancer 1999;7:121-7.

40 Zigmond AS, Snaith RP. The hospital anxiety and depression scale. Acta Psychiatr Scand 1983;67:361-70.

41 Bjelland I, Dahl AA, Haug TT, et al. The validity of the hospital anxiety and depression scale. An updated literature review. J Psychosom Res 2002;52:69-77.

42 LoMartire R, Äng BO, Gerdle B, et al. Psychometric properties of short Form-36 health survey, EuroQol 5-dimensions, and hospital anxiety and depression scale in patients with chronic pain. Pain 2020;161:83-95. 